\title{
Automated Discovery of Geometrical Theorems in GeoGebra
}

\author{
Zoltán Kovács \\ The Private University College of Education of the Diocese of Linz* \\ Linz, Austria \\ zoltan@geogebra.org
}

\author{
Jonathan $\mathrm{H}$. Yu \\ Gilman School \\ Baltimore, Maryland, USA \\ jonathanhy314@gmail.com
}

\begin{abstract}
We describe a prototype of a new experimental GeoGebra command and tool, Discover, that analyzes geometric figures for salient patterns, properties, and theorems. This tool is a basic implementation of automated discovery in elementary planar geometry. The paper focuses on the mathematical background of the implementation, as well as methods to avoid combinatorial explosion when storing the interesting properties of a geometric figure.
\end{abstract}

\section{Introduction}

In this paper we introduce a new GeoGebra command and tool Discover, which is available in the development GitHub repository ${ }^{1}$. This research is closely related to the former project Automated Geometer ${ }^{2}$ (see [1, 2, 3] for further details).

Given a Euclidean geometry construction drawn in GeoGebra, suppose a user wants to know if a given object $O$ has some "interesting features," such as relevant theorems or properties. This object can be a point, a line, a circle, or something else, although in the current implementation $O$ will always be a point contained in a given construction. Without any further user input, the Discover command will then analyze $O$ for its interesting and relevant features, and present them to the user as a list of formulas, as well as graphical illustrations.

For example, let $A B C$ an arbitrary triangle, and let $D$ and $E$ be the midpoints of $B C$ and $A C$, respectively (Fig. 1). Has $D$ any noteworthy features? Yes: $D E$ is parallel to $A B$, independent of the position of $A, B$ and $C$. Indeed, the command Discover $(D)$ confirms this observation with the output shown in

${ }^{*}$ The work was partially supported by the grant PID2020-113192GB-I00 from the Spanish MICINN.

${ }^{1}$ https://github.com/kovzol/geogebra-discovery

2 https://github.com/kovzol/ag

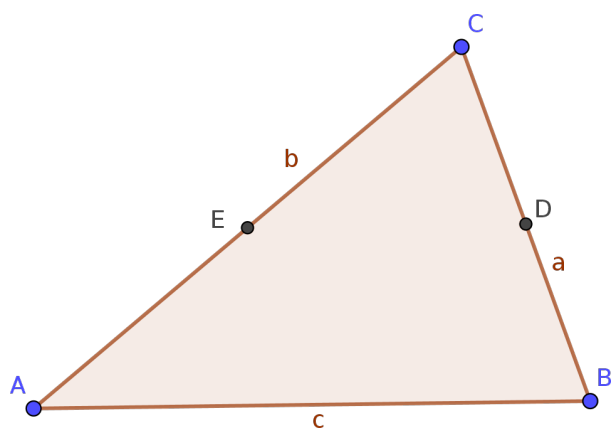

Figure 1: Initial setup for a discovery

J. Marcos, W. Neuper and P. Quaresma (Eds.): Theorem Proving Components for Educational Software 2021 (ThEdu'21)

EPTCS 354, 2022, pp. 1-12 doi 10.4204/EPTCS.354.1
(C) Kovács \& Yu

This work is licensed under the Creative Commons Attribution License. 


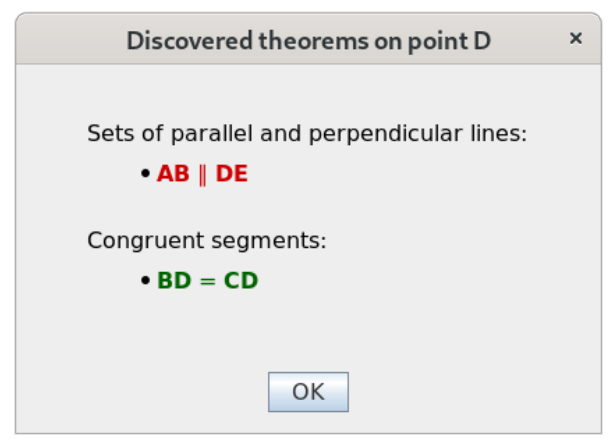

Figure 2: Output window of the Discover command that reports the Midline theorem

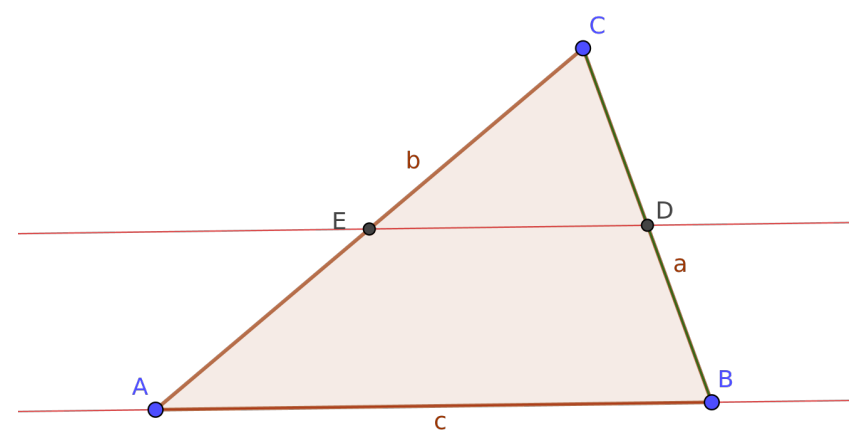

Figure 3: Further output of the Discover command

Fig. 23, GeoGebra adds lines $D E$ and $A B$ in the same color (Fig. 3). (Note, however, that the current implementation of GeoGebra does not report that $2 \cdot|D E|=|A B|$.) Also, the software reports the somewhat trivial finding that the segments $B D$ and $C D$ are congruent, with $B D$ and $C D$ highlighted in the same color. (The fact that " $A E$ and $C E$ are congruent" is not reported because these segments do not have a direct relationship with the point $D$.)

This output can be obtained by selecting the Discover tool in GeoGebra's toolbox, and then clicking on the point $D$. This functionality is currently implemented in both GeoGebra versions Classic 5 and 6 and will soon be incorporated into the official GeoGebra versions.

The Discover command calculates its outputs with the following algorithm: First, all points are analyzed to determine whether they are the same as another point. Second, all possible point triplets are examined for collinearity. Third, all possible subsets containing four points on the figure are checked for concyclicity. With knowledge of collinear points, separate lines can be uniquely defined, in order to determine whether they are parallel. Next, congruent segments can be identified by considering the pairs of all possible point pairs. Finally, perpendicular lines are identified. This combination of numerical and symbolic processes provides the results of the Discover command.

Our second example illustrates a more complicated problem. A regular hexagon $A B C D E F$ is given in Fig. 4. Point $G$ is defined as the intersection of $A D$ and $B E$. In addition, $H=B E \cap C F, I=A D \cap C F$. The points $G, H$ and $I$ may have trivial differences in their numerical representations, but in the geometrical 


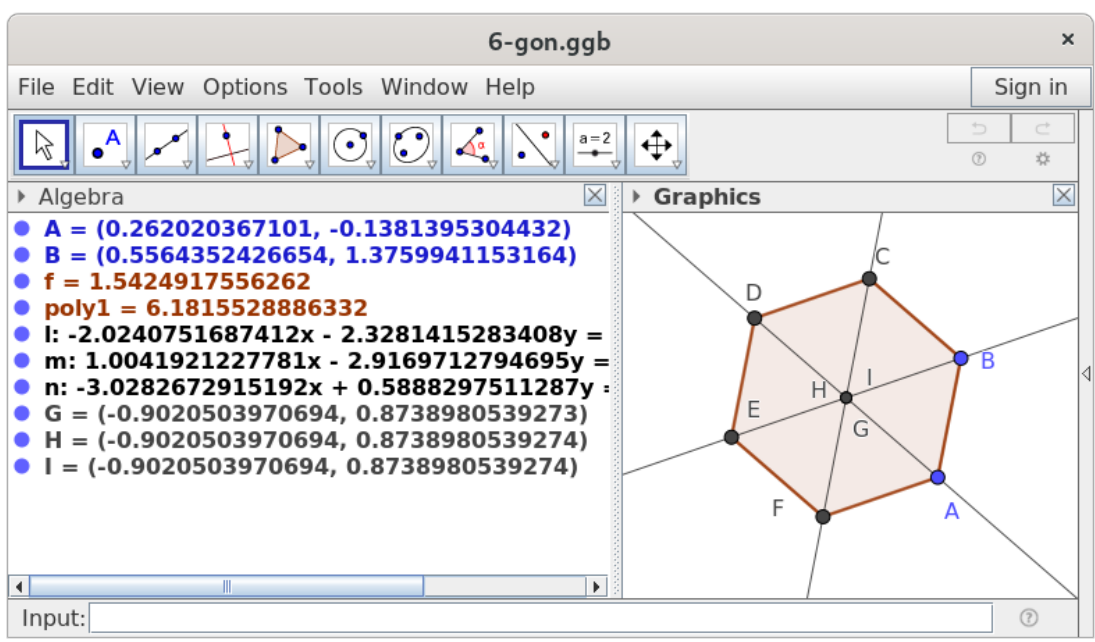

Figure 4: Initial setup for another discovery

sense they should be equal. For example, the $y$-coordinates of $G$ and $H$, as computed numerically by GeoGebra, differ only in the least significant decimal place shown. However, the final calculations to prove that they are identical will be symbolic and exact.

To determine any interesting features of point $F$, we enter the command Discover $(F)$. GeoGebra reports a set of properties in a message box and adds some additional outputs to the initial setup (Fig. 5).

Here we learn that the points $G, H$ and $I$ are all equal, as stated above. We also see that concylic points are reported as a single item, not as separate data. In addition, parallel line sets and their perpendicular supplementors are classified into three different sets, each colored with the same color. This allows the user to distinguish among the rectangular grids related to the objects of the figure. Finally, there are three sets of congruent segments. This approach in computation and reporting helps avoid combinatorial explosion.

\section{Mathematical background}

The above mentioned strategies have some similarities to the ones introduced in [4], but here we focus on minimizing the number of objects that have to be compared in the process that practically compares all objects with all other objects.

Our current implementation deals with points, lines, circles and parallel lines (or directions) and their perpendicular supplementors, and congruent segments.

A geometric point $P$ is a GeoGebra object, described by the GeoPoint class $\mathrm{s}^{3}$. While we will not provide a detailed definition of a geometric point, generally speaking, it is an object with a very complex structure containing two real coordinates, several style settings (including size and color, for example) and other technical details that are used in the application. Some geometric points are dependent of other geometric points or other geometric objects - this hierarchy is stored in the set of GeoPoints, too.

Independent of the detailed definition of a geometric point, we can still define the notion of point in our context.

\footnotetext{
${ }^{3}$ See GeoGebra's source code at github. com/geogebra/geogebra for more details.
} 

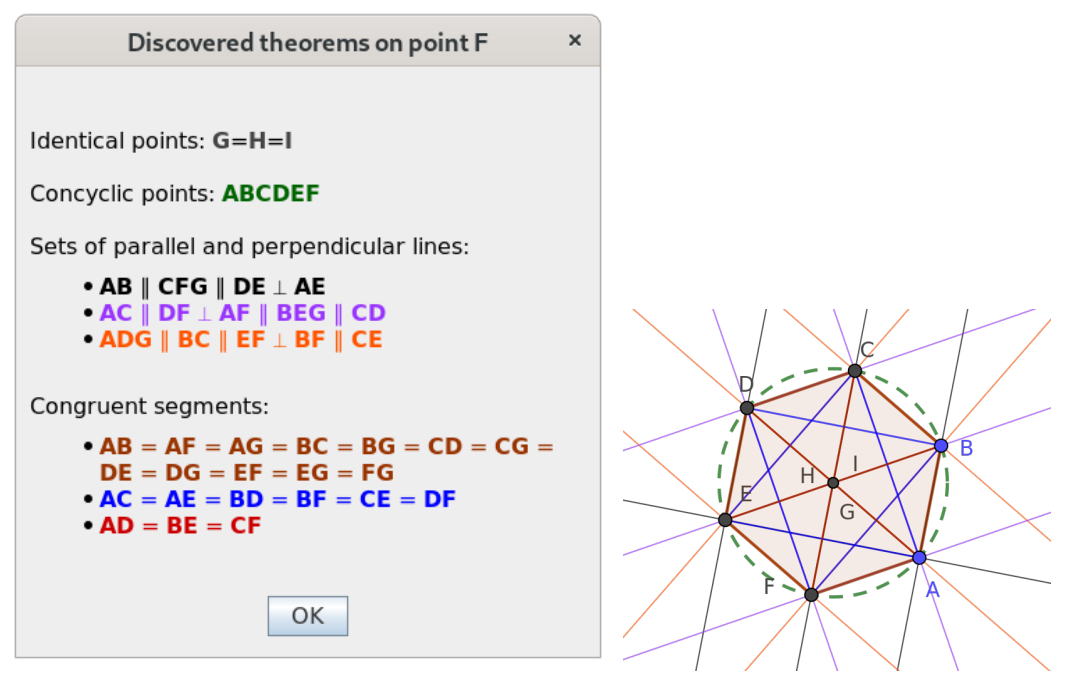

Figure 5: Output window that reports several theorems related to point $F$, and the geometric output of discovery

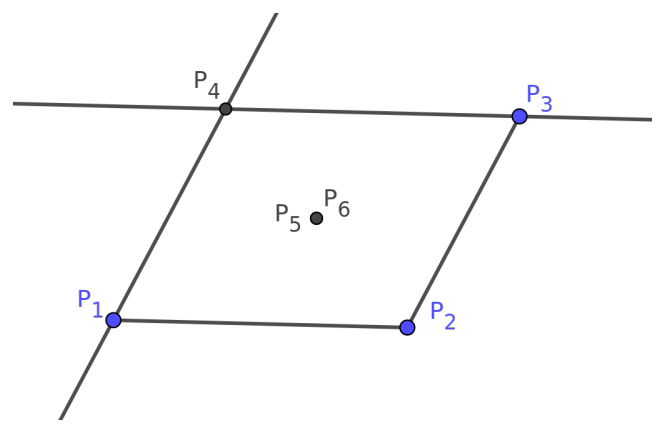

Figure 6: Points $P_{5}$ and $P_{6}$ are defined as midpoints of opposite vertices of parallelogram $P_{1} P_{2} P_{3} P_{4}$

Definition 1. A set of geometric points $\mathcal{P}=\left\{P_{1}, P_{2}, \ldots, P_{n}\right\}$ is called a point if for all different $P, Q \in \mathcal{P}$ the points $P$ and $Q$ are identical in general.

Henceforth, unless otherwise mentioned, we will consider points according to the definition above, not as geometric points.

Here, we do not precisely define when two points are identical in general. Instead, we will illustrate the concept of point identicality with the following example. Consider geometric points $P_{1}, P_{2}, P_{3}$ and $P_{4}$ that form a parallelogram. Now define $P_{5}$ and $P_{6}$ as the midpoint of $P_{1}$ and $P_{3}$, and $P_{2}$ and $P_{4}$, respectively. With these definitions, $P_{5}$ and $P_{6}$ are identical, because the diagonals of a parallelogram always bisect each other. In a dynamic geometry setting like GeoGebra, this simply means that by changing some points of the set $\left\{P_{1}, P_{2}, P_{3}, P_{4}\right\}$, the points $P_{5}$ and $P_{6}$ will still share the same position in the plane. (See Fig. 6. Here the construction is determined only by the points $P_{1}, P_{2}$ and $P_{3}$ : after they are freely chosen, the point $P_{4}$ must be dependent and uniquely defined as the intersection of the two parallel lines to $P_{1} P_{2}$ and $P_{2} P_{3}$, respectively, through $P_{3}$ and $P_{1}$.)

Statements considered "generally true" are true in most typical cases, but not $100 \%$ of the time, especially in cases of degenerate objects. For example, it is generally true that altitudes of a triangle meet at a point—but not always, since a degenerate triangle "usually" has three parallel "altitudes," 
unless two (or even three!) vertices of the triangle coincide. (See [5] for more details on the concept of general truth and degeneracies.)

Definition 2. A set of points $\ell=\left\{P_{1}, P_{2}, \ldots, P_{n}\right\}$ is called a line if for all different $P, Q, R \in \ell$ the points $P$, $Q$ and $R$ are collinear in general.

For example, the set $\ell=\{C, F, G\}$ in Fig. 5 forms a line.

Definition 3. A set of points $\mathcal{C}=\left\{P_{1}, P_{2}, P_{3}, \ldots, P_{n}\right\}$ is called a circle if for all different $P, Q, R, S \in \mathcal{C}$ the points $P, Q, R$ and $S$ are concyclic in general.

Definition 4. A set of lines $\vec{D}=\left\{\ell_{1}, \ldots, \ell_{n}\right\}$ is called parallel lines (or a direction) if for all different $\ell, m \in \vec{D}$ the lines $\ell$ and $m$ are parallel in general.

Definition 5. A pair of parallel lines $\vec{D}_{1}, \vec{D}_{2}$ is called perpendicular or parallel lines if for all different $\ell \in \vec{D}_{1}, m \in \vec{D}_{2}$ the lines $\ell$ and $m$ are parallel or perpendicular in general. We will use the notation $\vec{D}_{1} \perp \vec{D}_{2}$ in this case.

Definition 6. A set $\bar{s}=\{P, Q\}$ of two points is called a segment.

Definition 7. A set of segments $s=\left\{\overline{s_{1}}, \ldots, \overline{s_{n}}\right\}$ is called equal length segments (or congruent segments) if for all different $\overline{s_{1}}, \overline{s_{2}} \in s$ the segments $\overline{s_{1}}$ and $\overline{s_{1}}$ are equally long in general.

In fact, GeoGebra Discovery uses a more general concept of being identical: it allows two points (or two objects) to have a kind of relationship also if it is true just on parts (see [11] for more details).

The main idea of storing the objects is that points, lines, circles, directions (and their perpendicular supplementors) and equally long segments designate equivalence classes, that is:

Theorem 1. Let $\ell$ and $m$ be lines. Then, for all different points $P, Q, R \in m$, if $\{P, Q\} \subset \ell$, then $R \in \ell$; that $i s, \ell=m$.

Proof. In Euclidean geometry $\sqrt{4}$ two points always designate a unique line.

Theorem 2. Let $\mathcal{C}$ and $\mathcal{D}$ be circles. Then, for all different points $P, Q, R, S \in \mathcal{D}$, if $\{P, Q, R\} \subset \mathcal{C}$, then $S \in \mathcal{C}$; that is, $\mathcal{C}=\mathcal{D}$.

Proof. In Euclidean geometry three non-collinear points always designate a unique circle.

Theorem 3. Let $\vec{D}$ and $\vec{E}$ be directions. Let $\ell \in \vec{D}$ and $m \in \vec{E}$. If $\ell \| m$ in general, then $\vec{D}=\vec{E}$.

Proof. This follows immediately from the transitive property of parallelism.

Theorem 4. The relation $\perp$ is an equivalence relation on the parallel lines.

Proof. Reflexivity and symmetry are obvious. To check transitivity we assume that $\vec{D}_{1} \perp \vec{D}_{2}$ and $\vec{D}_{2} \perp \vec{D}_{3}$ hold and verify these four possible cases:

\begin{tabular}{c|c|c} 
& $\vec{D}_{2} \| \vec{D}_{3}$ & $\vec{D}_{2} \perp \vec{D}_{3}$ \\
\hline$\vec{D}_{1} \| \vec{D}_{2}$ & $\vec{D}_{1} \| \vec{D}_{3}$ & $\vec{D}_{1} \perp \vec{D}_{3}$ \\
\hline$\vec{D}_{1} \perp \vec{D}_{2}$ & $\vec{D}_{1} \perp \vec{D}_{3}$ & $\vec{D}_{1} \| \vec{D}_{3}$
\end{tabular}

We note that it is a requirement that all the lines are defined in the plane.

\footnotetext{
${ }^{4}$ The statement also holds in absolute geometry.
} 


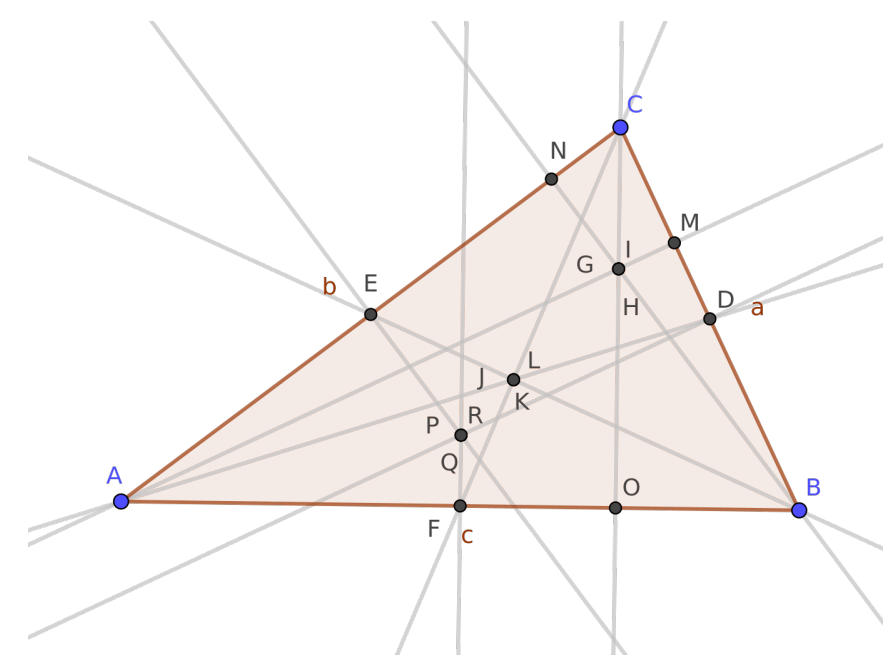

Figure 7: Euler line

Theorem 5. Let $s$ and t be segments. Let $\bar{u} \in s$ and $\bar{v} \in t$. If $|\bar{u}|=|\bar{v}|$ in general, then $s_{1}=s_{2}$.

Proof. This is an immediate consequence of the transitive property of equality of lengths.

By using these theorems we can maintain a minimal set of objects during discovery. See [12] for a more detailed description of the technical internals.

\section{Examples of Discover with selected theorems}

GeoGebra is a well-known and widely used software tool in education, with meaningful potential for using geometric discovery and exploration to teach elementary geometry. Even so, the range of mathematical knowledge is broad, including secondary school topics, international math competitions, and higher level mathematics. Below we examine selected theorems confirmed in the current implementation of Discover.

\subsection{Euler line}

The Euler line is a line determined from any triangle that is not regular. It passes through the orthocenter, the circumcenter and the centroid. The problem is shown in Fig. 7. With discovery on point $P$, the relevant theorems are listed in Fig. 8. The Euler line theorem implicitly includes several simple theorems, including concurrency of the altitudes $(G=H=I$, these points being the pairwise intersections of the altitudes), concurrency of the medians of a triangle $(J=K=L$, the generated points being the pairwise intersections of the medians), and concurrency of the perpendicular bisectors of the altitudes $(P=Q=R$, pairwise intersections as above).

\subsection{Nine-point circle}

The nine-point circle passes through nine significant points of an arbitrary triangle, namely:

- the midpoint of each side of the triangle, 


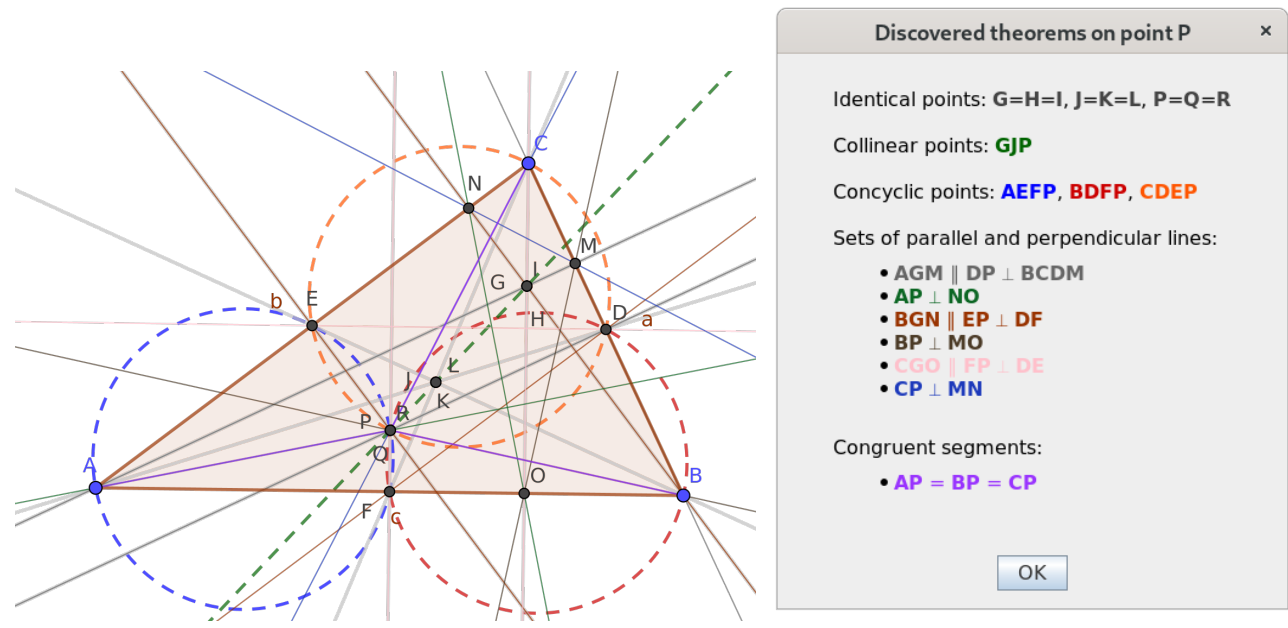

Figure 8: Output of the command Discover $(P)$

- the foot point of each altitude,

- the midpoint of the line segment from each vertex of the triangle to the orthocenter.

With discovery on the midpoint $D$ of side $B C$, the appropriate theorems are reported in Fig. 9. The nine-point circle theorem implicitly includes several other simple theorems. In addition, the graphical result suggests further theorems: segments $D J, E K$, and $F L$ are congruent and concurrent; these three segments are also diameters of the nine-point circle; and their intersection designates the center of the nine-point circle. This can be easily confirmed by performing another discovery.

\subsection{A contest problem}

In 2010, at the 51st International Mathematics Olympiad in Astana, Kazakhstan, the following shortlisted problem was proposed by United Kingdom:

Let $A B C$ be an acute triangle with $D, E, F$ the feet of the altitudes lying on $B C, C A, A B$ respectively. One of the intersection points of the line $E F$ and the circumcircle is $P$. The lines $B P$ and $D F$ meet at point $Q$. Prove that $A P=A Q$.

After constructing an acute triangle with GeoGebra Discovery (noting that no matching figure can be drawn for a non-acute input angle), we start discovery on point $Q$.

The discovered theorems appear in Fig. 10. We learn a few unexpected properties: $D P \| E Q$, and the points $C, D, P, Q$, and $A, F, P, Q$ are concyclic.

\section{Discussion}

\subsection{Trivial statements and theorems}

After the Discover command determines the salient properties for a given input, it displays relevant theorems but does not report trivial statements. One example of a trivial statement occurs in Fig. 2, in which the collinearity of points $B, C$ and $D$ and of points $A, C$ and $E$ are not reported. By defining $D$ as the midpoint of $B C$, this collinearity is implicitly assumed, so it does not make any sense to reiterate this. 


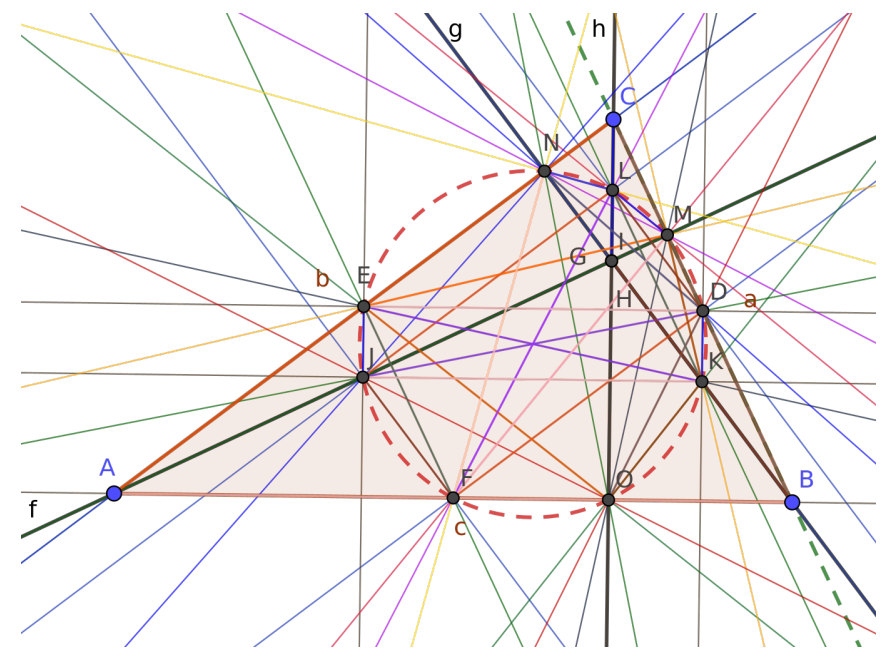

\begin{tabular}{|c|c|}
\hline Discovered theorems on point $D$ & $x$ \\
\hline \multicolumn{2}{|l|}{ Identical points: $\mathbf{G}=\mathbf{H}=$ I } \\
\hline \multicolumn{2}{|l|}{ Collinear points: BCDM } \\
\hline \multicolumn{2}{|l|}{ Concyclic points: DEFJKLMNO } \\
\hline \multicolumn{2}{|l|}{ Sets of parallel and perpendicular lines: } \\
\hline \multicolumn{2}{|l|}{ 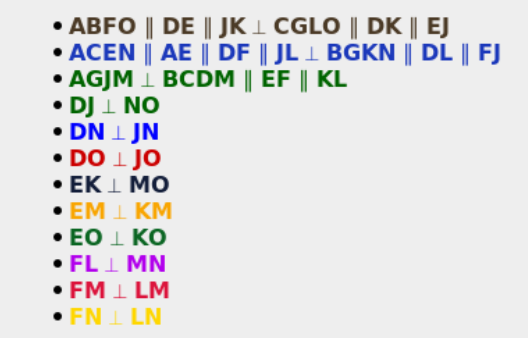 } \\
\hline \multicolumn{2}{|l|}{ Congruent segments: } \\
\hline \multirow{2}{*}{\multicolumn{2}{|c|}{ 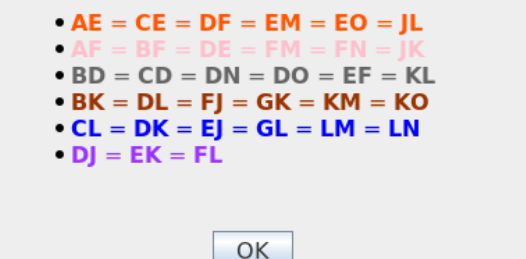 }} \\
\hline & \\
\hline
\end{tabular}

Figure 9: Output of the command Discover $(D)$
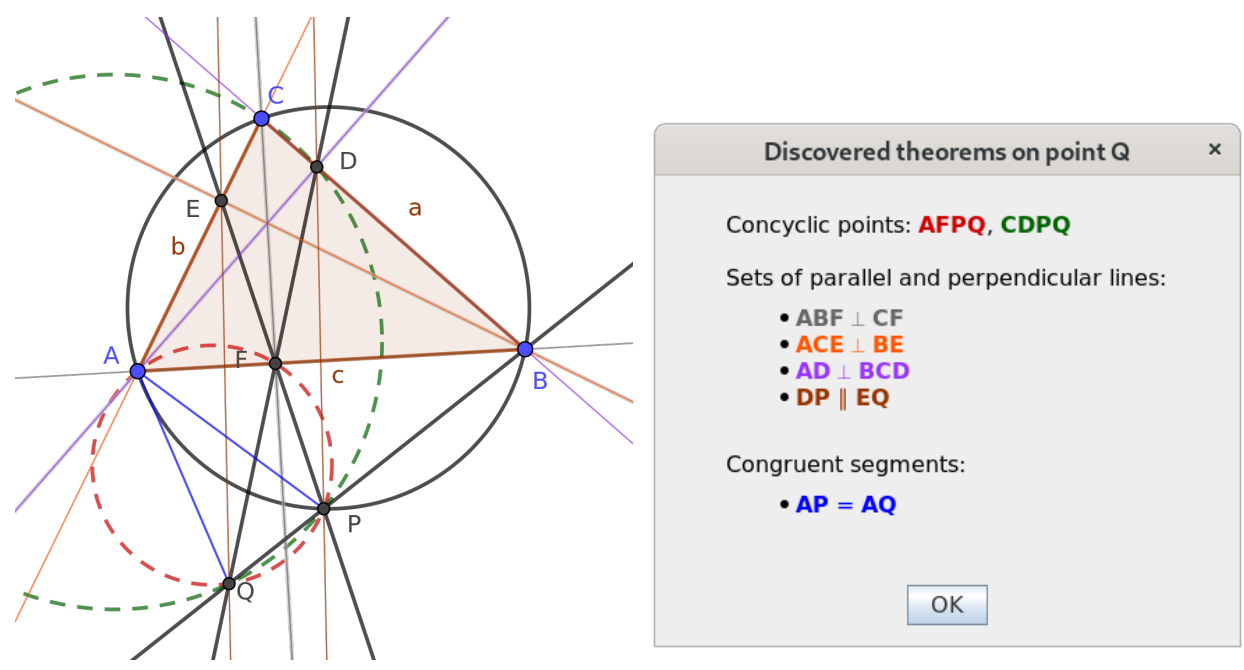

Figure 10: Output of the command Discover $(Q)$ 
The question of which properties are considered trivial or not has been a topic of long term discussion. Over 30 years ago, in his famous book, Larry Wos asked, "What properties can be identified to permit an automated reasoning program to find new and interesting theorems, as opposed to proving conjectured theorems?" [17]. This decision, at some level, does become a judgment call that may vary depending on the expertise of the user. Most users would regard the above statement $B D=C D$ as trivial, given that $D$ is the midpoint of $B C$. On the other hand, explicitly stating this property might be helpful for some beginners. Currently, GeoGebra Discovery regards as trivial all statements that directly follow from the parameters of the original problem, as demonstrated in the example above. However, more detailed criteria to determine which theorems to display could be clarified in the future. Recent research may help in crafting effective algorithms that prioritize identifying the most interesting theorems while filtering out less important statements (see [8, 15]).

\subsection{Combinatorial explosion and computational complexity}

By using the classes of the equivalence relations, the number of statements to be checked can be decreased significantly.

For each possible statement, a numerical check is first performed. Unfortunately, for some exotic coordinates of the points in the figure, the numerical check can be completely misleading. For example, very large numbers can sometimes result in numerically unstable computations. Regardless, if a numerical check is positive, then the statement is added to the list of conjectures, but if it is negative, no conjecture is registered. As a consequence, while our implementation may miss some true statements (due to numerical errors), it will not output false statements.

For each conjecture, a symbolic check will be performed. If the symbolic check is positive, then the statement will be saved as a theorem. If the symbolic check is negative, then the statement will be removed from the list of conjectures. If the symbolic check cannot determine whether a conjecture is true or false, the conjecture is removed from the list.

A special case of a conjecture is $P_{1}=P_{2}$ for each two geometric points. If this conjecture cannot be proven or disproven symbolically, then the discovery process will be halted. The user will then be notified that the construction must be redrawn in a different way, or else no output can be produced. This is required to maintain the consistency of the internal data.

Symbolic checks usually require more time than numerical verifications. The underlying computation uses Gröbner bases that require at most double exponential time of the number of variables [14] according to the algebraic translation of the given figure. Usually, the number of variables are double the number of geometric points in the figure (since there are two coordinates for each).

GeoGebra internally sets 5 seconds for the maximal execution time of each symbolic check. After timeout the result of the symbolic check will be undecided.

Complexity can be decreased by hiding some points in the figure. This allows for faster processing and avoids an overly cluttered output containing too much information.

\subsection{User interface enhancements}

Currently only points can be investigated. In a future version, a set of points, segments, lines, circles or a set of these could be allowed as input.

At the moment the computation process cannot be interrupted by the user. Given a large number of points in the figure, the calculation can be time consuming. For example, investigating the relationships 
of a regular 20-gon may require about 4 minutes on a modern personal computer (in our test a Lenovo ThinkPad E480 with an i7 processor, 16 GB RAM, Ubuntu Linux 18.04, was used).

The current version, which is based on GeoGebra Classic 5, performs better than the one on Classic 6. The latter is a web implementation of the GeoGebra application and uses a WebAssembly compilation of the computer algebra system Giac. Even if the code is reasonably fast as embedded code in a web page, Classic 6 underperforms the native technology: the same hardware is unable to handle the input of the regular 20-gon, with Google Chrome 83 crashing after 12 minutes of computation.

\subsection{Angles}

In a complex algebraic geometry setting, the study of angles is not as straightforward as investigating other objects. For a future version, however, this feature would be an important improvement.

By combining algebraic and pure geometric observations, however, simple theorems on angle equality could be easily detected. For example, Fig. 10 states that points $A, F, P, Q$ are concyclic. The inscribed angle theorem automatically implies $\angle Q A P=\angle Q F P$, among others.

\subsection{Stepwise suggestions}

Prior research (see [10, p. 46]) proposed that collecting the interesting new objects in a figure could be done stepwise, similarly to GeoGebra's former feature "special objects." For our midline theorem example (Fig. 1), this meant that after constructing the triangle $A B C$ and then midpoint $D$, the system automatically displayed the segments $B D$ and $C D$. The user could then accept these newly generated segments or remove them from the system. Then, by creating midpoint $E$, the system could show lines $A B$ and $D E$ to visualize parallelism.

However, this "special objects" feature was recently removed from GeoGebra after feedback that many users found this feature confusing. How stepwise suggestions might be implemented in GeoGebra Discovery remains a topic of future investigation.

\section{Related work}

We now discuss several projects that share some similarity to GeoGebra Discover but differ in significant ways.

First, GeoGebra Discovery is not the first tool that systematically displays confirmed theorems in a geometric figure. We refer the reader to

- Zlatan Magajna's OK Geometry [5],

- Jacques Gressier's Géométrix ${ }^{6}$ and

- Java Geometry Expert](JGEX) [18].

These systems are available free of charge, but the source code is available only for JGEX. On the other hand, GeoGebra Discovery focuses on an intuitive user interface and proofs in the most mathematical sense.

\footnotetext{
5 www.ok-geometry.com

6 geometrix.free.fr

7 https://github.com/yezheng1981/Java-Geometry-Expert
} 
Second, we note that there is a growing interest in creating algorithms related to success completion of secondary school or undergraduate mathematics entrance exams. (See [6, 16, 7], among others.) Sometimes these projects rely significantly on techniques used in the underlying computational methods. Also, these projects are often related to artificial intelligence and Big Data rather than to computational mathematics.

Third, we mention a theoretical issue. The idea to store a geometric point only once if it is identical to another one was previously described in Kortenkamp's work [9, 9.3.1]. This concept is a main design element in the dynamic geometry software Cinderella, which never stores a geometric point twice if the two variants are identical in general.

GeoGebra has a different design concept by allowing the user an arbitrary number of identical points to be defined. From the theorem prover's point of view, GeoGebra's concept is more difficult to handle, and a kind of translation is required to have a different data structure by using the concepts from Section 2.

Finally, we note that GeoGebra Discovery proves the truth in a different manner from Cinderella, with Cinderella using a probabilistic method, and GeoGebra Discovery literally proving all the deduced facts.

\section{Conclusion}

We described a prototype of the Discover command that is available in an experimental version of GeoGebra, called GeoGebra Discovery. This tool facilitates geometric analysis to determine important properties and theorems based on the user's input. Our current implementation can be directly downloaded from https://github.com/kovzol/geogebra/releases/tag/v5.0.641.0-2021Sep03

Active testing and sample outputs of the Discover command can be seen at https://prover-t est.geogebra.org/job/GeoGebra_Discovery-discovertest/. Currently, 19 test problems are successfully demonstrated, including Brahmagupta's theorem, Napoleon's theorem, Thebault's first two theorems, as well as some simpler statements. Areas for future development are listed in Section 4 .

\section{Acknowledgments}

The Discover command is a result of a long collaboration of several researchers. The project was initiated by Tomás Recio in 2010, and several other researchers joined, including Francisco Botana and M. Pilar Vélez, to name just the most prominent collaborators. The development and research work was continuously monitored and supported by the GeoGebra Team. Special thanks to Markus Hohenwarter, project director of GeoGebra.

\section{References}

[1] Francisco Botana, Zoltán Kovács \& Tomás Recio (2018): Automated Geometer, a web-based discovery tool. Presentation at ADG-12, Nanning, China, doi:10.13140/RG.2.2.19792.76807

[2] Francisco Botana, Zoltán Kovács \& Tomás Recio (2018): Towards an Automated Geometer. Presentation at AISC-13, Suzhou, China, doi $10.13140 /$ RG.2.2.36788.71042.

[3] Francisco Botana, Zoltán Kovács \& Tomás Recio (2018): Towards an Automated Geometer. In Jacques Fleuriot, Dongming Wang \& Jacques Calmet, editors: Artificial Intelligence and Symbolic Computation, Lecture 
Notes in Artificial Intelligence 11110, Springer International Publishing, pp. 215-220, doi 10.1007/978-3319-99956-2-15.

[4] Xiaoyu Chen, Dan Song \& Dongming Wang (2014): Automated generation of geometric theorems from images of diagrams. Annals of Mathematics and Artificial Intelligence 74(3-4), pp. 1-26, doi $10.1007 / \mathrm{s} 10472-$ 014-9433-7.

[5] Shang-Ching Chou (1987): Mechanical Geometry Theorem Proving. Springer Science + Business Media, doi $10.1007 / 978-94-009-4037-6$

[6] Hongguang Fu, Jingzhong Zhang, Xiuqin Zhong, Mingkai Zha \& Li Liu (2019): Robot for Mathematics College Entrance Examination. In: Electronic Proceedings of the 24th Asian Technology Conference in Mathematics, Mathematics and Technology, LLC.

[7] Akira Fujita, Akihiro Kameda, Ai Kawazoe \& Yusuke Miyao (2014): Overview of Todai robot project and evaluation framework of its NLP-based problem solving. In: Proceedings of the Ninth International Conference on Language Resources and Evaluation (LREC'14), pp. 2590-2597.

[8] Hongbiao Gao, Jianbin Li \& Jingde Cheng (2019): Measuring Interestingness of Theorems in Automated Theorem Finding by Forward Reasoning Based on Strong Relevant Logic. In: 2019 IEEE International Conference on Energy Internet (ICEI), pp. 356-361, doi:10.1109/ICEI.2019.00069.

[9] Ulrich Kortenkamp (1999): Foundations of Dynamic Geometry. Ph.D. thesis, ETH Zürich, doi:10.3929/ETHZ-A-003876663

[10] Zoltán Kovács (2019): Towards a new GeoGebra Geometry App. Presentation at MatemaTech Seminar for teachers, České Budějovice, Czechia, doi 10.13140/RG.2.2.25544.98568.

[11] Zoltán Kovács, Tomás Recio \& M. Pilar Vélez (2019): Detecting truth, just on parts. Revista Matemática Complutense 32, pp. 451-474, doi:10.1007/s13163-018-0286-1

[12] Zoltán Kovács \& Jonathan H. Yu (2020): Towards Automated Discovery of Geometrical Theorems in GeoGebra. CoRR abs/2007.12447. Available at https://arxiv.org/abs/2007.12447.

[13] Zlatan Magajna (2011): An observation tool as an aid for building proofs. The Electronic Journal of Mathematics and Technology 5(3), pp. 251-260.

[14] E.W. Mayr \& A.R. Meyer (1982): The Complexity of the Word Problem for Commutative Semigroups and Polynomial Ideals. Advances in Mathematics 46, pp. 305-329, doi:10.1016/0001-8708(82)90048-2

[15] Y. Puzis, Y. Gao \& G. Sutcliffe (2006): Automated generation of interesting theorems. In G. Sutcliffe \& R. Goebel, editors: Proceedings of the 19th International FLAIRS Conference, AAAI Press, Menlo Park, pp. 49-54.

[16] Minjoon Seo, Hannaneh Hajishirzi, Ali Farhadi, Oren Etzioni \& Clint Malcolm (2015): Solving Geometry Problems: Combining Text and Diagram Interpretation. In: Proceedings of the 2015 Conference on Empirical Methods in Natural Language Processing, pp. 1466-1476, doi:10.18653/v1/D15-1171

[17] Larry Wos (1988): Automated Reasoning: 33 Basic Research Problems. Prentice-Hall.

[18] Zheng Ye, Shang-Ching Chou \& Xiao-Shan Gao (2011): An Introduction to Java Geometry Expert. In: Automated Deduction in Geometry, Springer Science + Business Media, pp. 189-195, doi:10.1007/978-3642-21046-4_10 